\title{
Vitamin C enhances the expression of IL17 in a Jmjd2-dependent manner
}

\author{
Mi Hye Song, Varun Sasidharan Nair \& Kwon Ik Oh ${ }^{*}$ \\ Department of Pathology, Hallym University College of Medicine, Chuncheon 24252, Korea
}

\begin{abstract}
Previously, we reported that vitamin $\mathrm{C}$ facilitates the $\mathrm{CpG}$ demethylation of Foxp3 enhancer in $\mathrm{CD}^{+}{ }^{+} \mathrm{Foxp}^{+}$regulatory T cells (Tregs) by enhancing the activity of a DNA demethylase ten-eleven-translocation (Tet). However, it is not clear whether vitamin $C$ affects other helper $T$ cell lineages like $T$ helper type 17 (Th17) cells which are related with Tregs. Here, we show that the expression of interleukin-17A (IL17) increases with the treatment of vitamin $\mathrm{C}$ but not with other antioxidants. Interestingly, the upregulation of IL17 was not accompanied by DNA demethylation in $/ 117$ promoter and was independent of Tet enzymes. Rather, vitamin $\mathrm{C}$ reduced the trimethylation of histone $\mathrm{H} 3$ lysine 9 ( $\mathrm{H} 3 \mathrm{~K} 9 \mathrm{me} 3)$ in the regulatory elements of the $I I 17$ locus, and the effects of vitamin $C$ were abrogated by knockdown of jumonji-C domain-containing protein 2 (jmj22). These results suggest that vitamin $C$ can affect the expression of IL17 by modulating the histone demethylase activity. [BMB Reports 2017; 50(1): 49-54]
\end{abstract}

\section{INTRODUCTION}

T helper type 17 (Th17) cells are important cellular mediators in diverse immune responses (1). The generation of the Th17 cell is best achieved by stimulation with TCR, CD28 and cytokines like interleukin-6 (IL6) and transforming growth factor- $\beta$ (TGF- $\beta$ ) (2). TCR/CD28 signals activate pioneering transcription factors to open up and make the target gene chromatin structure permissive to cytokine-associated transcription factor (3), signal transducer and activator of transcription 3 (STAT3). Then, STAT3 acts together with various modifiers to promote the expression of the gene encoding retinoic acid receptor-related orphan receptor $\gamma t$ (RORYt; encoded by Rorc) $(4,5)$ leading to the lineage specification of Th17 cells. Th17 cells produce a group of

*Corresponding author. Tel: +82-33-248-2564; Fax: +82-33-2418250; E-mail: kwonik@hallym.ac.kr

https://doi.org/10.5483/BMBRep.2017.50.1.193

Received 21 November 2016, Revised 1 December 2016, Accepted 8 December 2016

Keywords: H3K9me3, Histone modification, IL17, Jmjd2, Vitamin C proinflammatory cytokines including IL17, which mediate diverse autoimmune and inflammatory diseases (6).

Vitamin $C$ is known to be not only a general anti-oxidant but also a cofactor for a large enzyme family known as the ironand 2-oxoglutarate-dependent dioxygenases (7). A typical example is the collagen prolyl-4-hydroxylase in collagen maturation and scurvy (8). Recently, we found that ten-eleventranslocation (Tet) DNA dioxygenase also works in a vitamin C dependent manner in regulatory $T$ cells (Tregs). In the absence of vitamin $\mathrm{C}$, the initial hydroxylation of 5-methylcytosine by Tet enzymes proceeded albeit less efficiently; however, the intermediates, 5-hydroxymethylcytosine, accumulated gradually, and a further oxidation reaction was not completed (9). Collectively, vitamin $\mathrm{C}$ seems to have a role in reducing inactive iron and maintaining continued enzyme cycling in DNA oxidation followed by demethylation reactions.

In this study, based on our previous report showing the role of vitamin C in Tregs, we investigated Th17 cells after vitamin $\mathrm{C}$ treatment and found that vitamin $\mathrm{C}$ exploited Jmjd2 histone demethylases to enhance the expression of IL17.

\section{RESULTS AND DISCUSSION}

\section{IL17 was upregulated by vitamin C treatment}

To examine the role of vitamin C in Th17 cell differentiation, we sorted naïve $\mathrm{CD}^{+}{ }^{+} \mathrm{T}$ cells from wild type (WT) mice and primed them to differentiate into Th17 in the presence or absence of vitamin C $(10 \mu \mathrm{g} / \mathrm{ml})$. After $3 \mathrm{~d}$, we restimulated the cells with PMA and ionomycin and assessed the expression of cytokines by intracellular staining and flow cytometry. Unexpectedly, in the Th17 polarizing condition (anti-CD3/CD28 mAbs plus rlL6 and TGF- $\beta$ ), some vitamin $\mathrm{C}$ - treated cells, but not control cells, differentiated into Foxp3-expressing induced Tregs, which remained up to $5 \mathrm{~d}$ (Supplementary Fig. 1). Previously, vitamin $\mathrm{C}$ was reported to induce the demethylation of $\mathrm{CpG}$ motifs in the Foxp3 enhancer and contribute to the stable expression of Foxp3 in Tregs $(9,10)$, which led us to hypothesize that vitamin $\mathrm{C}$ stabilized the expression of Foxp3 even in the presence of rIL6. Indeed, the CpG motifs in the Foxp3 enhancer regions were demethylated when vitamin $\mathrm{C}$ was available. These findings led us to modify the Th17 differentiation condition by adding anti-IL2 blocking mAbs (clone: S4B6), which prevented the Foxp3 expression

ISSN: 1976-670X (electronic edition)

Copyright (c) 2017 by the The Korean Society for Biochemistry and Molecular Biology

(c) This is an open-access article distributed under the terms of the Creative Commons Attribution Non-Commercial License (http://creativecommons.org/licenses/by-nc/4.0) which permits unrestricted non-commercial use, distribution, and reproduction in any medium, provided the original work is properly cited. 
(Supplemental Fig. 1) and enabled us to analyze the effect of vitamin C on Th17 cell development.

FACS-sorted naïve $\mathrm{CD}^{+}{ }^{+} \mathrm{T}$ cells were cultured again under the modified Th17 conditions in the presence or absence of vitamin C. Interestingly, IL17- expressing cells were generated more efficiently from cells treated with vitamin C (Fig. 1A). To check the specificity, we performed Th1 differentiation experiments in parallel and found that vitamin $\mathrm{C}$ did not influence the expression of IFN- $\gamma$ under Th1 conditions (Fig. 1B). The mRNA level of $/ / 17$ was also significantly increased in the vitamin $\mathrm{C}$-treated Th17 cells. However, the level of Rorc transcripts was transiently elevated during an early stage (Fig.

A

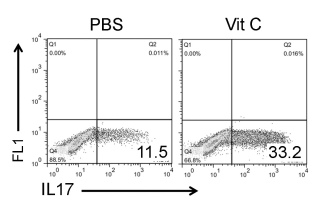

C

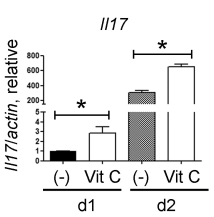

D

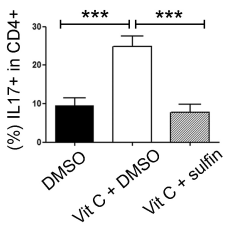

B
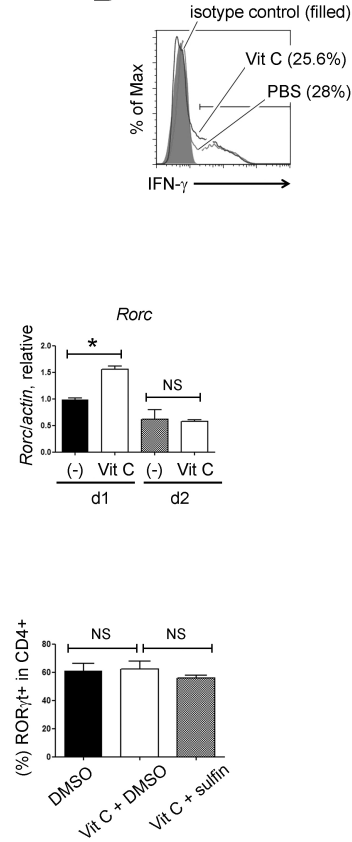

Fig. 1. Vitamin $C$ enhances the expression of IL17. (A) FACSsorted naïve $\mathrm{CD}^{+}{ }^{+} \mathrm{T}$ cells were cultured in the presence or absence of vitamin $C(10 \mu \mathrm{g} / \mathrm{ml})$ under Th17 polarizing condition for $3 \mathrm{~d}$ and IL17 expression was checked by flow cytometry after PMAVionomycin restimulation. Data are representative of three independent experiments. (B) FACS-sorted naïve $\mathrm{CD}^{+}{ }^{+} \mathrm{T}$ cells were cultured in the presence or absence of vitamin $C(10 \mu \mathrm{g} / \mathrm{ml})$ under Th1 polarizing condition and IFN- $\gamma$ expression was checked by flow cytometry. Data are representative of two independent experiments. (C) Th17 cells were generated in the presence or absence of vitamin $\mathrm{C}$ and then, the expression levels of the indicated gene transcripts were checked by RT-qPCR (mean \pm SEM of duplicates, from one experiment representative of three independent experiments). (D) Percentages of IL17 (left) or ROR $\gamma$ t (right)-expressing cells cultured under Th17 conditions in the presence of vitamin $C \pm$ sulfinpyrazone (sulifin). Bars show the mean \pm SEM of the results $(n=6-8)$ pooled from three independent experiments. FACS plots of (A) and (B) refer to the percentage of each subset. $* * * P<0.001 ; * P<0.05 ; N S$, not significant.

1C). We confirmed the effect of vitamin $C$ once again with a sodium-dependent vitamin $\mathrm{C}$ transporter inhibitor, sulfinpyrazone (11). Sulfinpyrazone treatment reversed the effect of vitamin C on IL17 expression (Fig. 1D, left) suggesting that the entry of vitamin C is essential. FACS analysis also showed that the frequency of ROR $\gamma \mathrm{t}-$ expressing cells was not significantly altered by the vitamin $\mathrm{C}$ or sulfinpyrazone treatment (Fig. 1D, right). To examine the mechanism of action of vitamin $\mathrm{C}$, we checked whether other anti-oxidants could also increase the expression of IL17. Naïve $\mathrm{CD}^{+}{ }^{+} \mathrm{T}$ cells were treated with vitamin $\mathrm{C}$, glutathione $(\mathrm{GSH}, 5 \mathrm{mM})$, or dithiothreitol (DTT, $0.3 \mathrm{mM}$ ) under the Th17 polarizing conditions for $3 \mathrm{~d}$, and neither GSH nor DTT increased the expression of IL17 (Supplementary Fig. 2A). Altogether, these findings suggest that the effect of vitamin C on IL17 expression was independent of ROR $\gamma t$ upregulation and its role as a general reducer.

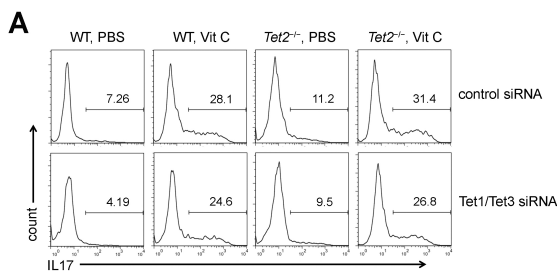

B

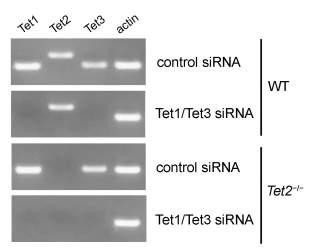

C

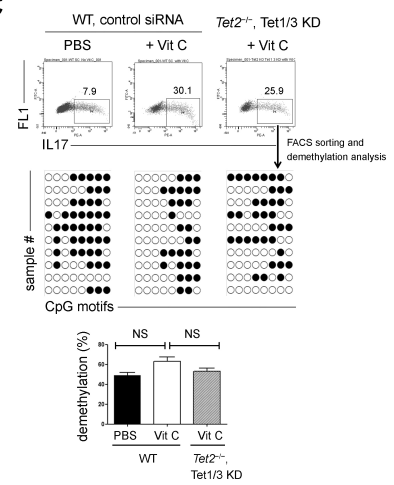

Fig. 2. Tet proteins were not essential for vitamin $\mathrm{C}$-induced IL17 expression. (A) FACS-sorted $\mathrm{WT}$ and $\mathrm{Tet} 2^{-1-}$ naïve $\mathrm{CD} 4^{+} \mathrm{T}$ cells were transfected with control or Tet1/Tet3 siRNAs and cultured under the indicated conditions. IL17 expression was analyzed by flow cytometry. (B) FACS-sorted WT and Tet2 ${ }^{-/}$ naïve $\mathrm{CD}^{+}{ }^{+} \mathrm{T}$ cells were transfected with Tet1/Tet3 siRNAs, and the expression of the indicated gene transcripts was checked by RT-PCR. (C) FACS-sorted WT and Tet2 $2^{-1-}$ naïve $\mathrm{CD}^{+} \mathrm{T}$ cells were transfected with control or Tet1/Tet3 siRNAs and cultured under the indicated conditions. IL $17^{+}$cells were re-sorted and subjected to bisulfate sequencing. Results of statistical analysis are shown as the mean \pm SEM of the results $(n=3)$ pooled from two independent experiments. Numbers in the FACS plots of (A) and $(C)$ refer to the percentage of each subset. Methylation status of individual CpG motif was shown by white (demethylation) or black (methylation) circles. 
Tet proteins were not required for the vitamin $\mathrm{C}$-induced IL17 upregulation

Because vitamin $\mathrm{C}$ has a role as a cofactor for iron and 2-oxoglutarate dependent dioxygenases including DNA demethylases (Tet family) (12), histone demethylases (jumonji domain-containing proteins, the Jmjd family) (13) and prolyl hydroxylase domain-containing proteins (PHD proteins) (14), we decided to investigate whether these pathways are involved in the vitamin C-induced IL17 upregulation. First, we examined whether PHD proteins and related hypoxiainducible factor pathways (15) are involved by using $\mathrm{N}$-oxalylglycine (NOG), a potent inhibitor of PHD proteins (16-18). We cultured naïve $\mathrm{CD}^{+} \mathrm{T}$ cells under Th17 conditions in the presence of vitamin $\mathrm{C}$ plus various doses of NOG and found that NOG treatment enhances, not inhibits, Th17 differentiation (Supplementary Fig. 2B) consistent with a previous report showing the positive effects of hypoxiainducible factor on IL17 expression (19).

It was reported that the CpG motifs in the IL17 promoter are less methylated in Th17 cells (20), and 5-hydroxymethylcytosine and Tet2 have roles in the generation and functions of Th17 cells (21), which led us to hypothesize that vitamin C might contribute to IL17 expression as a cofactor of Tet2. To address this issue, we cultured $\mathrm{WT}$ and $T e t 2^{-1-}$ naïve $\mathrm{CD} 4^{+} \mathrm{T}$ cells with vitamin $C$ under Th17 conditions and examined the effect of vitamin C. Unexpectedly, the expression of IL17 was increased by vitamin C in Tet $2^{-1-}$ cells as well (Fig. 2A). Given the potential redundancy between members of the Tet family (22), we investigated whether Tet 1 and Tet 3 functioned as substitutes for Tet2. WT and Tet $2^{-1-}$ naïve $\mathrm{CD} 4^{+} \mathrm{T}$ cells were co-transfected with equal amounts of small interference RNAs (siRNAs) targeting Tet 1 and Tet3 and cultured under Th17 conditions in the presence of vitamin C. RT-PCR assays revealed that Tet 1 and Tet 3 transcripts were specifically downregulated in the Tet1/Tet3 siRNA-transfected cells (Fig. 2B). After $3 d$, the expression level of IL17 was analyzed by flow cytometry. IL17 was not altered significantly by the knockdown of Tet1 and Tet3 (Fig. 2A).

Next, the methylation status of the CpG motifs in the IL17 promoter was analyzed. FACS-sorted $\mathrm{WT}$ and $\mathrm{Tet} 2^{-1-}$ naïve $\mathrm{CD}^{+}{ }^{+} \mathrm{T}$ cells were transfected with control or Tet1/Tet3 siRNAs, cultured under Th17 conditions, and restimulated with PMA and ionomycin. Then, $\mathrm{IL}_{1} 7^{+}$cells were re-sorted and subjected to bisulfite sequencing. The extent of $\mathrm{CpG}$ demethylation was not significantly changed by vitamin C (Fig. 2C) suggesting that vitamin C facilitated IL17 expression in a Tet-independent manner.

\section{The effects of vitamin $\mathrm{C}$ were abrogated by the knockdown of Jmjd2 histone demethylases}

Next, we tested repressive histone modifications like trimethylation of histone $\mathrm{H} 3$ lysine $9(\mathrm{H} 3 \mathrm{~K} 9 \mathrm{me} 3)$ or histone $\mathrm{H} 3$ lysine 27 (H3K27me3) and the related Jmjd histone demethylases. Because the roles of H3K27me3 demethylase, a
A
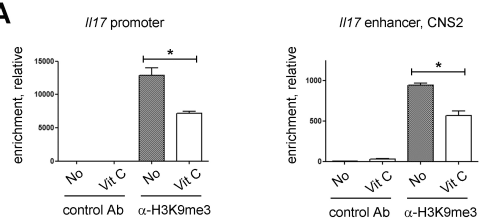

B

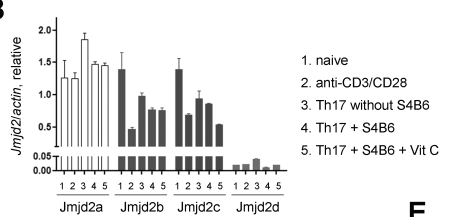

C
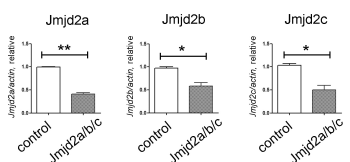

E
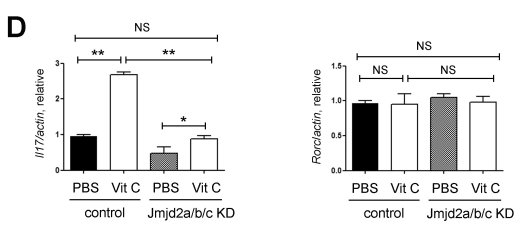

$\mathbf{F}$

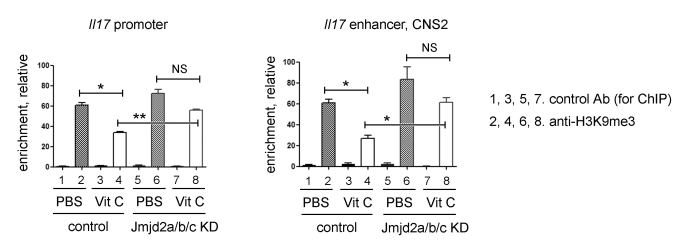

Fig. 3. Vitamin $\mathrm{C}$ worked in a Jmjd2 dependent manner. (A) ChIP-qPCR of H3K9me3 at the IL17 promoter and enhancer (CNS2) locus. FACS-sorted naïve $\mathrm{CD}^{+}{ }^{+} \mathrm{T}$ cells were cultured under Th17 conditions in the presence or absence of vitamin $C$ and used for the ChIP analysis. Subsequent qPCR was done on DNA precipitated by control and anti-H3K9me3 Ab (mean \pm SEM of duplicates, from one experiment representative of three independent experiments). (B) Quantitative RT-PCR was performed to assess the expression levels of Jmjd2 members in the indicated $\mathrm{CD}^{+} \mathrm{T}$ cells. (C) FACS-sorted naïve $\mathrm{CD}^{+} \mathrm{T}$ cells were transfected with control or Jmjd2a/b/c siRNAs, and then, the expressions of the indicated gene transcripts were checked by RT-qPCR. (D) The expression levels of $1 / 17$ (left) and Rorc (right) transcripts were investigated in the indicated Th17 cell populations. (E) IL17 expressions in the indicated Th17 cell populations were checked by flow cytometry. (F) ChIP-qPCR of H3K9me3 at the IL17 promoter and enhancer (CNS2) locus. FACS-sorted naïve CD4 ${ }^{+} \mathrm{T}$ cells were cultured under Th17 conditions in the presence or absence of vitamin $C$ and used for the ChIP analysis. Subsequent qPCR was done on DNA precipitated by control and antiH3K9me3 Ab (mean + SEM of duplicates, from one experiment representative of three independent experiments). Data are representative of $3(\mathrm{~A}, \mathrm{C}-\mathrm{E})$ or $2(\mathrm{~B}, \mathrm{~F})$ independent experiments. $* * P<0.01 ; * P<0.05 ; N S$, not significant. 
Jmjd3, in Th17 differentiation were controversial $(23,24)$ and vitamin $\mathrm{C}$ was reported to induce the expression of pluripotent genes in induced stem cells (iPSCs) by erasing the methylation of H3K9 (13), we decided to focus on the changes of $\mathrm{H} 3 \mathrm{~K} 9 \mathrm{me} 3$ at the IL17 locus (25). As shown in Fig. 3A, vitamin $\mathrm{C}$ treatment reduced $\mathrm{H} 3 \mathrm{~K} 9 \mathrm{me} 3$ in the IL17 promoter and enhancer (CNS2) indicating that H3K9 is one of the sites for chromatin modification in response to vitamin $\mathrm{C}$. Because demethylation at $\mathrm{H} 3 \mathrm{~K} 9 \mathrm{me} 3$ is catalyzed by the histone demethylase Jmjd2 subfamily (26) and three (Jmjd2a, Jmjd2b, and Jmjd2c) out of four Jmjd2 members are expressed in various types of $\mathrm{CD}^{+}{ }^{+} \mathrm{T}$ cells (Fig. 3B), we tried to knock down three Jmjd2 members (referred to as Jmjd2a/b/c hereafter) together using siRNAs to prevent potential redundancy among them (27) and investigated whether vitamin C works in a Jmjd2-dependent manner. Jmjd2a/b/C transcripts were significantly downregulated after siRNA transfection (Fig. 3C). WT naïve $\mathrm{CD}^{+}{ }^{+}$T cells transfected with control or $\mathrm{Jmjd} 2 \mathrm{a} / \mathrm{b} / \mathrm{c}$ siRNA were cultured under Th17 polarizing conditions in the presence or absence of vitamin $\mathrm{C}$. Subsequent RT-qPCR (Fig. 3D, left) and FACS analysis (Fig. 3E) revealed that the effect of vitamin $\mathrm{C}$ on the expression of IL17 was impaired, albeit partially, by the knockdown of Jmjd2a/b/c. In contrast to IL17, the expression of Rorc transcripts was not altered (Fig. 3D, right). Our findings imply that vitamin C enhances IL17 expression by modulating Jmjd2. To examine the H3K9me3 enrichments in the $/ 117$ locus of Th17 cells treated with vitamin $C$ or Jmjd2a/b/c siRNA, we performed the chromatin immunoprecipitation (ChIP) assay. As shown previously (Fig. 3A), the enrichment of H3K9me3 was reduced by vitamin $\mathrm{C}$ in the $/ 117$ regulatory elements (promoter and enhancer) in the Th17 cells transfected with control siRNA. However, the effect of vitamin $\mathrm{C}$ on H3K9me3 was lost after the treatment with the Jmjd2a/b/c siRNAs (Fig. 3F).

Altogether, these findings indicate that vitamin $\mathrm{C}$ induced the upregulation of IL17 through Jmjd2 histone demethylase enzymes and the related H3K9 histone modifications. Further study on Jmjd2 enzymes and vitamin C will advance our understanding on the regulatory mechanisms of Th17 development and provide new therapeutic targets for Th17-related diseases.

\section{MATERIALS AND METHODS}

\section{Mice}

WT C57BL/6 mice were purchased from Koatech (Pyeongtaek, Gyeongi-do, Korea). Floxed Tet2 transgenic (B6;129S-Tet $2^{\text {tmi.11aai } / \text { J, }}$ Tet $2^{\mathrm{fl} / \mathrm{fl}}$ ) mice were obtained from The Jackson Laboratory (Bar Harbor, ME). To generate Tet2 deficient mice (referred to as Tet $2^{-l-}$ mice), CD4-Cre transgenic mice were crossed to floxed Tet2 transgenic mice. All animal experimentations were conducted in accordance with guidelines and approval of the International Animal Care and Use Committees of Hallym University (Hallym 2015-63).

\section{Cell isolation and flow cytometry}

To sort naïve $\mathrm{CD}^{+}$cells, CD44 and CD25 were used $\left(\mathrm{CD} 4{ }^{+} \mathrm{CD} 8{ }^{-} \mathrm{CD} 44^{\text {low }} \mathrm{CD} 25^{-}\right)$. The post-sort purity for each cell type was usually $>97 \%$. Intracellular cytokines were stained using Foxp3 Staining Buffer set (eBioscience, San Diego, CA). For cytokine analysis, cells were cultured for $4 \mathrm{~h}$ in the presence of PMA/ionomycin plus monensin (BD biosciences, San Jose, CA) before intracellular cytokine staining (28). Data were acquired through FACS Calibur or FACS Canto-II (BD Biosciences) and were analyzed with Flowlo software (Tree Star, Ashland, OR).

\section{Cell culture}

For Th17 cell generation, cells were stimulated by platebound anti-CD3 (2C11, $5 \mu \mathrm{g} / \mathrm{ml}$; eBioscience) plus CD28 (37.51, $1 \mu \mathrm{g} / \mathrm{ml}$; eBioscience), recombinant murine IL6 (rIL6, $20 \mathrm{ng} / \mathrm{ml}$; Peprotech, Rocky Hill, NJ) and recombinant TGF- $\beta$ (rTGF- $\beta, 0.5 \mathrm{ng} / \mathrm{ml}$; Peprotech) in the presence or absence of vitamin C (10 $\mu \mathrm{g} / \mathrm{ml}$, Sigma-Aldrich, St. Louis, MO) for $3 \mathrm{~d}$. To prevent the Foxp3 expression, $10 \mu \mathrm{g} / \mathrm{ml}$ of anti-IL2 blocking $\mathrm{mAb}$ (clone: S4B6) was always added except some samples in supplementary Fig. 1.

\section{Small interfering RNA transfection}

Transfection was performed using the Amaxa Mouse T Cell Nucleofector Kit and the Nucleofector device (X-001 program) according to the previous report (29). Cells were transfected with 300 pmol SMART pool siRNAs (Dharmacon, Lafayette, Colorado) designed against mouse Jmjd2a (M-059020-00-0005), Jmjd2b (M-062955-00-0005) and Jmjd2c (M-051504-00-0005) together. Non-targeting control siRNAs (SN-1001, Bioneer) were also transfected as control treatments. The expression levels of each target molecule were checked $8 \mathrm{~h}$ after electroporation.

\section{Quantitative PCR (qPCR)}

PCR reactions were performed on RotorGene 6000 system (Qiagen, Valencia, CA) using AccuPower GreenStar qPCR kit (Bioneer, Seoul, Korea). All data were normalized to actin. Non-specific amplification was checked by using melting curves and agarose gel electorphoresis (30). The sequences of primers are as below.

Mouse Jmjd2a-Forward: 5'-GACCACACTCTGCCCACAC-3' Mouse Jmjd2a-Reverse: 5'-TCCTGGGGTATTTCCAGACA-3' Mouse Jmjd2b-Forward: 5'-GGCTTTAACTGCGCTGAGTC-3' Mouse /mjd2b-Reverse: 5'-GTGTGGTCCAGCACTGTGAG-3' Mouse Imjd2c-Forward: 5'-CACGGAGGACATGGATCTCT-3' Mouse Jmjd2c-Reverse: 5'-CGAAGGGAATGCCATACTTC-3' Mouse Actin-Forward: 5'-CATCCGTAAAGACCTCTATGCC AAC-3'

Mouse Actin-Reverse: 5'-ATGGAGCCACCGATCCACA-3'

\section{DNA demethylation analysis}

FACS-sorted $\mathrm{IL}_{17} 7^{+}$cells were incubated with $300 \mu \mathrm{l}$ lysis 
buffer (10 mM Tris-HCl, $100 \mathrm{mM} \mathrm{NaCl,} 50 \mathrm{mM}$ EDTA, 0.5\% SDS, $0.1 \mu \mathrm{g} / \mathrm{ml}$ proteinase $\mathrm{K}$, and $20 \mu \mathrm{g} / \mathrm{ml}$ RNase A) for $24 \mathrm{~h}$ at $60^{\circ} \mathrm{C}$. Then extracted genomic DNAs were converted by the EZ DNA methylation gold kit (Zymo Research, Irvine, CA) and subjected to PCR (sense: 5'-ACAAATTCATAAACCCCAACAA CTA-3', antisense: 5'-TGTTGATTTTATTTGAGGATGGAAT-3'). The PCR products were cloned into the pGemT-easy vector (Promega, Madison, WI) and individual clones were sequenced with M13 reverse primer (GAAACAGCTATGACCATG).

\section{Chromatin immunoprecipitation (ChIP)}

Nuclear lysate was sonicated to make small DNA fragments ranging from 100-500 base pairs and then incubated with anti-H3K9me3 (MABI 0319, Active motif, Carlsbad, CA) overnight at $4^{\circ} \mathrm{C}$. Isotype-matched control $\mathrm{Ab}$ was used for the negative control. Immune complexes containing DNA fragments were precipitated using EZ-ChIP kit (Milipore, Darmstadt, Germany). Relative enrichment of the target regions in the precipitated DNA fragments was analyzed by qPCR. The sequences of primers are as follows.

I/17 promoter forward, 5'-GCAGCAGCTTCAGATATGTCC-3' I/17 promoter reverse, 5'-TGAGGTCAGCACAGAACCAC-3' II17 CNS2 enhancer forward, 5'-CCGTTTAGACTTGAAACC CAGTC-3'

II17 CNS2 enhancer reverse, 5'-GTACCTATGTGTTAGGAG GCGC-3'

\section{Statistical analyses}

A two-tailed, unpaired, Student's t-test was used to calculate the statistical significance of differences between groups. $\mathrm{P}$ values are represented as follows: ${ }^{* * * P}<0.001 ; * * \mathrm{P}<0.01$; ${ }^{*} \mathrm{P}<0.05$, whereas NS, not significant, is used to denote $\mathrm{P}$ values $>0.05$. Error bars indicate s.e.m.

\section{ACKNOWLEDGEMENTS}

This research was supported by Basic Science Research Program through the National Research Foundation of Korea (NRF) funded by the Ministry of Education (NRF-2016R1D 1A1B03934212) and Hallym University (HRF-201612-016).

\section{CONFLICTS OF INTEREST}

The authors have no conflicting financial interests.

\section{REFERENCES}

1. Korn T, Bettelli E, Oukka M and Kuchroo VK (2009) IL-17 and Th17 Cells. Annu Rev Immunol 27, 485-517

2. Mangan PR, Harrington LE, O'Quinn DB et al (2006) Transforming growth factor-beta induces development of the $\mathrm{T}(\mathrm{H}) 17$ lineage. Nature 441, 231-234

3. Ciofani M, Madar A, Galan C et al (2012) A validated regulatory network for Th17 cell specification. Cell 151, 289-303
4. Durant L, Watford WT, Ramos HL et al (2010) Diverse targets of the transcription factor STAT3 contribute to T cell pathogenicity and homeostasis. Immunity 32, 605-615

5. Ivanov II, McKenzie BS, Zhou L et al (2006) The orphan nuclear receptor RORgammat directs the differentiation program of proinflammatory IL-17+ T helper cells. Cell 126, 1121-1133

6. Patel DD and Kuchroo VK (2015) Th17 Cell Pathway in Human Immunity: Lessons from Genetics and Therapeutic Interventions. Immunity 43, 1040-1051.

7. Young JI, Zuchner S and Wang G (2015) Regulation of the Epigenome by Vitamin C. Annu Rev Nutr 35, 545-564

8. Gorres KL and Raines RT (2010) Prolyl 4-hydroxylase. Crit Rev Biochem Mol Biol 45, 106-124

9. Sasidharan Nair V, Song MH and Oh KI (2016) Vitamin C Facilitates Demethylation of the Foxp3 Enhancer in a Tet-Dependent Manner. J Immunol 196, 2119-2131

10. Yue X, Trifari S, Aijo T et al (2016) Control of Foxp3 stability through modulation of TET activity. J Exp Med 213, 377-397

11. Dickson KM, Gustafson CB, Young JI, Zuchner S and Wang G (2013) Ascorbate-induced generation of 5hydroxymethylcytosine is unaffected by varying levels of iron and 2-oxoglutarate. Biochem Biophys Res Commun 439, 522-527

12. Xu GL and Walsh CP (2014) Enzymatic DNA oxidation: mechanisms and biological significance. BMB Rep 47, 609-618

13. Chen J, Liu H, Liu J et al (2013) H3K9 methylation is a barrier during somatic cell reprogramming into iPSCs. Nat Genet 45, 34-42

14. Knowles HJ, Raval RR, Harris AL and Ratcliffe PJ (2003) Effect of ascorbate on the activity of hypoxia-inducible factor in cancer cells. Cancer Res 63, 1764-1768

15. Baek SH and Kim KI (2016) Regulation of HIF-1alpha stability by lysine methylation. BMB Rep 49, 245-246

16. Hopkinson RJ, Tumber A, Yapp C et al (2013) 5-Carboxy8-hydroxyquinoline is a Broad Spectrum 2-Oxoglutarate Oxygenase Inhibitor which Causes Iron Translocation. Chem Sci 4, 3110-3117

17. Schofield CJ and Ratcliffe PJ (2004) Oxygen sensing by HIF hydroxylases. Nat Rev Mol Cell Biol 5, 343-354

18. Chowdhury R, Yeoh KK, Tian YM et al (2011) The oncometabolite 2-hydroxyglutarate inhibits histone lysine demethylases. EMBO Rep 12, 463-469

19. Dang EV, Barbi J, Yang HY et al (2011) Control of $\mathrm{T}(\mathrm{H}) 17 / \mathrm{T}$ (reg) balance by hypoxia-inducible factor 1 . Cell $146,772-784$

20. Yang BH, Floess S, Hagemann S et al (2015) Development of a unique epigenetic signature during in vivo Th17 differentiation. Nucleic Acids Res 43, 1537-1548

21. Ichiyama K, Chen T, Wang X et al (2015) The methylcytosine dioxygenase Tet2 promotes DNA demethylation and activation of cytokine gene expression in $\mathrm{T}$ cells. Immunity 42, 613-626

22. Blaschke K, Ebata KT, Karimi MM et al (2013) Vitamin C induces Tet-dependent DNA demethylation and a blastocyst-like state in ES cells. Nature 500, 222-226

23. Li Q, Zou J, Wang M et al (2014) Critical role of histone 
demethylase Jmjd3 in the regulation of CD4+ T-cell differentiation. Nat Commun 5, 5780

24. Liu Z, Cao W, Xu L et al (2015) The histone H3 lysine-27 demethylase Jmjd3 plays a critical role in specific regulation of Th17 cell differentiation. J Mol Cell Biol 7, 505-516

25. Xiao X, Shi X, Fan Y et al (2016) The Costimulatory Receptor OX40 Inhibits Interleukin-17 Expression through Activation of Repressive Chromatin Remodeling Pathways. Immunity 44, 1271-1283

26. Hojfeldt JW, Agger K and Helin K (2013) Histone lysine demethylases as targets for anticancer therapy. Nat Rev Drug Discov 12, 917-930

27. Pedersen MT, Kooistra SM, Radzisheuskaya A et al (2016)
Continual removal of $\mathrm{H} 3 \mathrm{~K} 9$ promoter methylation by Jmjd2 demethylases is vital for ESC self-renewal and early development. EMBO J 35, 1550-1564

28. Seong MA, Woo JK, Kang JH et al (2015) Oral administration of fermented wild ginseng ameliorates DSS-induced acute colitis by inhibiting NF-kappaB signaling and protects intestinal epithelial barrier. BMB Rep 48, 419-425

29. Mantei A, Rutz S, Janke M et al (2008) siRNA stabilization prolongs gene knockdown in primary $\mathrm{T}$ lymphocytes. Eur J Immunol 38, 2616-2625

30. Zuo J, Ma H, Cai H, Wu Y, Jiang $W$ and $Y u L$ (2015) An inhibitory role of NEK6 in TGFbeta/Smad signaling pathway. BMB Rep 48, 473-478 